\title{
SOME LIMIT THEOREMS CONNECTED WITH BROWNIAN LOCAL TIME
}

\author{
RAOUF GHOMRASNI
}

Received 26 October 2004; Revised 11 April 2005; Accepted 12 April 2005

Let $B=\left(B_{t}\right)_{t \geq 0}$ be a standard Brownian motion and let $\left(L_{t}^{x} ; t \geq 0, x \in \mathbb{R}\right)$ be a continuous version of its local time process. We show that the following $\operatorname{limit}_{\lim }(1 / 2 \varepsilon) \int_{0}^{t}\left\{F\left(s, B_{s}-\right.\right.$ $\left.\varepsilon)-F\left(s, B_{s}+\varepsilon\right)\right\} d s$ is well defined for a large class of functions $F(t, x)$, and moreover we connect it with the integration with respect to local time $L_{t}^{x}$. We give an illustrative example of the nonlinearity of the integration with respect to local time in the random case.

Copyright (c) 2006 Raouf Ghomrasni. This is an open access article distributed under the Creative Commons Attribution License, which permits unrestricted use, distribution, and reproduction in any medium, provided the original work is properly cited.

\section{Introduction}

1.1. The local time of the Brownian motion $B$ at the point $a$ is defined as follows:

$$
L_{t}^{a}=\mathbb{P} \lim _{\varepsilon \downarrow 0} \frac{1}{2 \varepsilon} \int_{0}^{t} 1_{\left(\left|B_{s}-a\right| \leq \varepsilon\right)} d s
$$

which equivalently could be written as follows:

$$
L_{t}^{a}=\mathbb{P} \lim _{\varepsilon \downarrow 0} \frac{1}{2 \varepsilon} \int_{0}^{t}\left(1_{\left(B_{s}-\varepsilon \leq a\right)}-1_{\left(B_{s}+\varepsilon \leq a\right)}\right) d s .
$$

Here we are, more generally, interested in the limit in $L^{1}$ :

$$
\lim _{\varepsilon \downarrow 0} \frac{1}{2 \varepsilon} \int_{0}^{t}\left\{F\left(s, B_{s}-\varepsilon\right)-F\left(s, B_{s}+\varepsilon\right)\right\} d s
$$

for some function $F$.

Our motivation comes from the desire to connect Chitashvili and Mania results [1] with those of Eisenbaum [2]. 
2 Some limit theorems connected with Brownian local time

1.2. We give an example which illustrates that the integration with respect to $\left(L_{t}^{x} ; 0 \leq t \leq\right.$ $1, x \in \mathbb{R}$ ) does not admit a linear extension in the random case (see Section 3.2 for details) and in particular local time is not a 1-integrator, which is also proved by Eisenbaum [2].

\section{Notation and preliminaries}

Let $B=\left(B_{t}\right)_{t \geq 0}$ be a standard Brownian motion and let $\left(L_{t}^{x} ; t \geq 0, x \in \mathbb{R}\right)$ be a continuous version of its local time process. Let $\left(\mathscr{F}_{t}\right)_{t \geq 0}$ denote the natural filtration generated by $B$. Without loss of generality, we restrict our attention to functions defined on $[0,1] \times \mathbb{R}$.

For a measurable function $f$ from $[0,1] \times \mathbb{R}$ into $\mathbb{R}$, define the norm $\|\cdot\|$ by

$$
\|f\|=2\left(\int_{0}^{1} \int_{\mathbb{R}} f^{2}(s, x) e^{-x^{2} / 2 s} \frac{d s d x}{\sqrt{2 \pi s}}\right)^{1 / 2}+\int_{0}^{1} \int_{\mathbb{R}}|x f(s, x)| e^{-x^{2} / 2 s} \frac{d s d x}{s \sqrt{2 \pi s}} .
$$

Let $\mathscr{H}$ be the set of functions $f$ such that $\|f\|<\infty$.

In Eisenbaum [2], it is shown that the integration with respect to $L$ is possible in the following sense. Let $f_{\Delta}$ be an elementary function on $[0,1] \times \mathbb{R}$, meaning that

$$
f_{\Delta}(t, x)=\sum_{\left(s_{i}, x_{j}\right) \in \Delta} f_{i, j} 1_{\left(s_{i}, s_{i+1}\right]}(t) 1_{\left(x_{j}, x_{j+1}\right]}(x)
$$

where $\Delta=\left\{\left(s_{i}, x_{j}\right), 1 \leq i \leq n, 1 \leq j \leq m\right\}$ is an $[0,1] \times \mathbb{R}$ grid, and, for every $(i, j), f_{i j}$ is in $\mathbb{R}$. For such a function, integration with respect to $L$ is defined by

$$
\int_{0}^{1} \int_{\mathbb{R}} f_{\Delta}(s, x) d L_{s}^{x}=\sum_{\left(s_{i}, x_{j}\right) \in \Delta} f_{i, j}\left(L_{s_{i+1}}^{x_{j+1}}-L_{s_{i}}^{x_{j+1}}-L_{s_{i+1}}^{x_{j}}+L_{s_{i}}^{x_{j}}\right) .
$$

Let $f$ be an element of $\mathscr{H}$. For any sequence of elementary functions $\left(f_{\Delta_{k}}\right)_{k \in \mathbb{N}}$ converging to $f$ in $\mathscr{H}$, the sequence $\left(\int_{0}^{1} \int_{\mathbb{R}} f_{\Delta_{k}}(s, x) d L_{s}^{x}\right)_{k \in \mathbb{N}}$ converges in $L^{1}$. The limit obtained does not depend on the choice of the sequence $\left(f_{\Delta_{k}}\right)$ and represents the integral $\int_{0}^{1} \int_{\mathbb{R}} f(s, x) d L_{s}^{x}$.

Theorem 2.1 (see $[2])$. Let $(A(x, t) ; x \in \mathbb{R}, 0 \leq t \leq 1)$ be a continuous random process taking values in $\mathbb{R}$, such that for any $t$ in $[0,1]$ and any $\omega, A(\cdot, t)$ is absolutely continuous with respect to $d x$. Note $\partial A / \partial x$ its derivative and ask $\partial A / \partial x$ to be continuous. Then $\int_{0}^{1} \int_{\mathbb{R}} A(x, s) d L_{s}^{x}$ exists and the following hold:

(i) for any couple $(a, b)$ in $\mathbb{R}^{2}$ with $a<b$

$$
\int_{0}^{t} \int_{b}^{a} A(x, s) d L_{s}^{x}=-\int_{0}^{t} \frac{\partial A}{\partial x}\left(B_{s}, s\right) d s+\int_{0}^{t} A(b, s) d_{s} L_{s}^{b}-\int_{0}^{t} A(a, s) d_{s} L_{s}^{a} ;
$$

(ii)

$$
\int_{0}^{1} \int_{\mathbb{R}} A(x, s) d L_{s}^{x}=-\int_{0}^{1} \frac{\partial A}{\partial x}\left(B_{s}, s\right) d s
$$

(iii)

$$
\left(\int_{0}^{t} \int_{b}^{a} A(x, s) d L_{s}^{x}\right)(\omega)=\int_{0}^{t} \int_{b}^{a} A(x, s)(\omega) d L_{s}^{x}(\omega)
$$




\section{Main results}

\subsection{Deterministic case}

Theorem 3.1. Let $F$ be a bounded element of $\mathcal{H}$. The following equalities hold in $L^{1}$ :

$$
\begin{aligned}
& \lim _{\varepsilon \downarrow 0} \frac{1}{\varepsilon} \int_{0}^{t}\left\{F\left(s, B_{s}\right)-F\left(s, B_{s}-\varepsilon\right)\right\} d s=-\int_{0}^{t} \int_{\mathbb{R}} F(s, x) d L_{s}^{x} ; \\
& \lim _{\varepsilon \downarrow 0} \frac{1}{\varepsilon} \int_{0}^{t}\left\{F\left(s, B_{s}+\varepsilon\right)-F\left(s, B_{s}\right)\right\} d s=-\int_{0}^{t} \int_{\mathbb{R}} F(s, x) d L_{s}^{x} ; \\
& \lim _{\varepsilon \downarrow 0} \frac{1}{2 \varepsilon} \int_{0}^{t}\left\{F\left(s, B_{s}-\varepsilon\right)-F\left(s, B_{s}+\varepsilon\right)\right\} d s=\int_{0}^{t} \int_{\mathbb{R}} F(s, x) d L_{s}^{x} .
\end{aligned}
$$

Remark 3.2. (1) If we take $F(t, x)=1_{(x \leq a)}$ in (3.1), we have the very definition of $L_{t}^{a}$.

(2) Eisenbaum [2] has shown that for any Borelian function $b(t)$,

$$
\lim _{\varepsilon \downarrow 0} \frac{1}{2 \varepsilon} \int_{0}^{t} 1_{\left(\left|B_{s}-b(s)\right|<\varepsilon\right)} d s=\int_{0}^{t} \int_{\mathbb{R}} 1_{(-\infty, b(s))}(x) d L_{s}^{x} \quad \text { in } L^{1},
$$

which corresponds to (3.3) with $F(t, x)=1_{(x \leq b(t))}$.

Proof. Define $H_{\mathcal{\varepsilon}}(t, x)=(1 / \varepsilon) \int_{x-\varepsilon}^{x} F(t, y) d y$. Then $H_{\varepsilon} \rightarrow F$ in $\mathscr{H}$ as $\varepsilon \downarrow 0$. On the one hand, $(\partial / \partial x) H_{\varepsilon}(t, x)=(1 / \varepsilon)\{F(t, x)-F(t, x-\varepsilon)\}$. It follows that (see Eisenbaum [2, Theorem 5.1(ii)]) $\int_{0}^{t} \int_{\mathbb{R}} H_{\varepsilon}(s, x) d L_{s}^{x}=-(1 / \varepsilon) \int_{0}^{t}\left\{F\left(s, B_{s}\right)-F\left(s, B_{s}-\varepsilon\right)\right\} d s$. On the other hand, $\int_{0}^{t} \int_{\mathbb{R}} H_{\varepsilon}(s$, $x) d L_{s}^{x} \rightarrow \int_{0}^{t} \int_{\mathbb{R}} F(s, x) d L_{s}^{x}$ in $L^{1}$.

Corollary 3.3 (see [3]). The following relation holds in $L^{1}$ :

$$
\lim _{\varepsilon \downarrow 0} \frac{1}{2 \varepsilon} \int_{0}^{t} g(s) I\left(b(s)-\varepsilon<B_{s}<b(s)+\varepsilon\right) d s=\int_{0}^{t} g(s) d L_{s}^{b}
$$

for a continuous function $g:[0, t] \rightarrow \mathbb{R}$ and a continuous curve $b(\cdot)$ with bounded variation on $[0, t]$.

Proof. We apply Theorem 3.1 to the function $F(t, x)=g(t) I(x<b(t))$. It follows that (1/ $2 \varepsilon) \int_{0}^{t} g(s) I\left(b(s)-\varepsilon<B_{s}<b(s)+\varepsilon\right) d s \rightarrow \int_{0}^{t} \int_{\mathbb{R}} g(s) I(x<b(s)) d L_{s}^{x}$ in $L^{1}$ as $\varepsilon \downarrow 0$. We conclude using (see [4, Corollary 2.9]) that for the continuous function $g$, we have $\int_{0}^{t} g(s) \partial_{s} L_{s}^{b(s)}=$ $\int_{0}^{t} g(s) d L_{s}^{b}$.

3.2. Random function case. Let $a, b$ be in $\mathbb{R}$ with $a<b$. Let $M$ be the set of elementary processes $A$ such that

$$
A(s, x)=\sum_{\left(s_{i}, x_{j}\right) \in \Delta} A_{i j} 1_{\left.s_{i}, s_{i+1}\right]}(s) 1_{\left(x_{j}, x_{j+1}\right]}(x),
$$

where $\left(s_{i}\right)_{1 \leq i \leq n}$ is a subdivision of $(0,1],\left(x_{j}\right)_{1 \leq j \leq m}$ is a finite sequence of real numbers in $(a, b], \Delta=\left\{\left(s_{i}, x_{j}\right), 1 \leq i \leq n, 1 \leq j \leq m\right\}$, and, is $A_{i j}$ an $\mathscr{F}_{s_{j}}$-measurable random variable such that $\left|A_{i j}\right| \leq 1$ for every $(i, j)$. 
4 Some limit theorems connected with Brownian local time

Eisenbaum [2] asked the following question: does integration with respect to $\left(L_{t}^{x} ; 0 \leq\right.$ $t \leq 1, x \in \mathbb{R}$ ) admit a linear extension to $\mathscr{P}$ the field generated by $\mathcal{M}$, verifying the following property?

If $\left(A_{n}\right)_{n \geq 0}$ converges a.e. to $A(t, x)$, then $\left(\int_{0}^{1} \int_{a}^{b} A_{n}(s, x) d L_{s}^{x}\right)_{n \geq 0}$ converges in $L^{1}$ to $\int_{0}^{1} \int_{a}^{b} A(s, x) d L_{s}^{x}$.

She only obtained a negative answer to the following weaker question:

$$
\text { Is the set }\left\{\int_{0}^{1} \int_{a}^{b} A(s, x) d L_{s}^{x}, A \in M\right\} \text { bounded in } L^{1} \text { ? }
$$

Consequently, integration with respect to $\left(L_{t}^{x} ; 0 \leq t \leq 1, x \in \mathbb{R}\right)$ does not admit a continuous extension in $L^{1}$.

Here we give an illustrative example, thanks to a result obtained by Walsh, which shows the lack of a linear extension.

Let us define $A_{\varepsilon}(t, x)=(1 / \varepsilon) \int_{x-\varepsilon}^{x} L_{t}^{y} d y$ and $\tilde{A}_{\varepsilon}(t, x)=(1 / \varepsilon) \int_{x}^{x+\varepsilon} L_{t}^{y} d y$. We see easily that $A_{\varepsilon}(t, x)$ (resp., $\left.\tilde{A}_{\varepsilon}(t, x)\right)$ converges a.e. to $L_{t}^{x}$, nevertheless we have

$$
\lim _{\varepsilon \downarrow 0} \int_{0}^{t} \int_{\mathbb{R}} A_{\varepsilon}(s, x) d L_{s}^{x} \neq \lim _{\varepsilon \downarrow 0} \int_{0}^{t} \int_{\mathbb{R}} \tilde{A}_{\varepsilon}(s, x) d L_{s}^{x} .
$$

Remark 3.4. The integrals $\int_{0}^{t} \int_{\mathbb{R}} A_{\varepsilon}(s, x) d L_{s}^{x}$ and $\int_{0}^{t} \int_{\mathbb{R}} \widetilde{A}_{\varepsilon}(s, x) d L_{s}^{x}$ are well defined thanks to Theorem 2.1, however, one does not know whether $\int_{0}^{t} \int_{\mathbb{R}} L_{s}^{x} d L_{s}^{x}$ is well defined or not.

Let us recall, for the convenience of the reader, Walsh's theorem about the decomposition of $A\left(t, B_{t}\right):=\int_{0}^{t} 1_{\left\{B_{s} \leq B_{t}\right\}} d s$.

Theorem 3.5 (see $[5])$. A $\left(t, B_{t}\right)$ has the decomposition

$$
A\left(t, B_{t}\right)=\int_{0}^{t} L_{s}^{B_{s}} d B_{s}+X_{t}
$$

where

$$
X_{t}=\lim _{\varepsilon \downarrow 0} \frac{1}{2 \varepsilon} \int_{0}^{t}\left\{L_{s}^{B_{s}}-L_{s}^{B_{s}-\varepsilon}\right\} d s=t+\lim _{\varepsilon \downarrow 0} \frac{1}{2 \varepsilon} \int_{0}^{t}\left\{L_{s}^{B_{s}+\varepsilon}-L_{s}^{B_{s}}\right\} d s .
$$

The limits exist in probability, uniformly for t in compact sets.

Our example follows by recalling the following property:

$$
\int_{0}^{t} \int_{\mathbb{R}} A_{\mathcal{\varepsilon}}(s, x) d L_{s}^{x}=-\frac{1}{\varepsilon} \int_{0}^{t}\left\{L_{s}^{B_{s}}-L_{s}^{B_{s}-\varepsilon}\right\} d s .
$$

\section{References}

[1] R. J. Chitashvili and M. G. Mania, Decomposition of the maximum of semimartingales and generalized Itô's formula, New Trends in Probability and Statistics, Vol. 1 (Bakuriani, 1990), VSP, Utrecht, 1991, pp. 301-350.

[2] N. Eisenbaum, Integration with respect to local time, Potential Analysis 13 (2000), no. 4, 303328. 
[3] G. Peskir, A change-of-variable formula with local time on curves, Research Report 428, Department of Theoretical Statistics, University of Aarhus, Aarhus, 2002, 17 pp.

[4] Ph. Protter and J. San Martín, General change of variable formulas for semimartingales in one and finite dimensions, Probability Theory and Related Fields 97 (1993), no. 3, 363-381.

[5] J. B. Walsh, Some remarks on $A\left(t, B_{t}\right)$, Séminaire de Probabilités 27, Lecture Notes in Mathematics, vol. 1557, Springer, Berlin, 1993, pp. 173-176.

Raouf Ghomrasni: Fakultät II-Mathematik und Naturwissenschaften, Institut für Mathematik, Technische Universität Berlin, Straße des 17. Juni 136, 10623 Berlin, Germany

E-mail address: ghomrasni@math.tu-berlin.de 


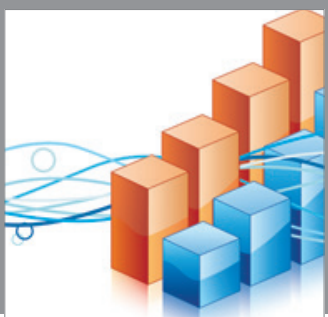

Advances in

Operations Research

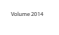

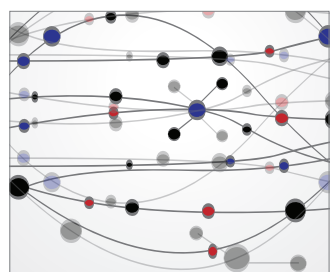

\section{The Scientific} World Journal
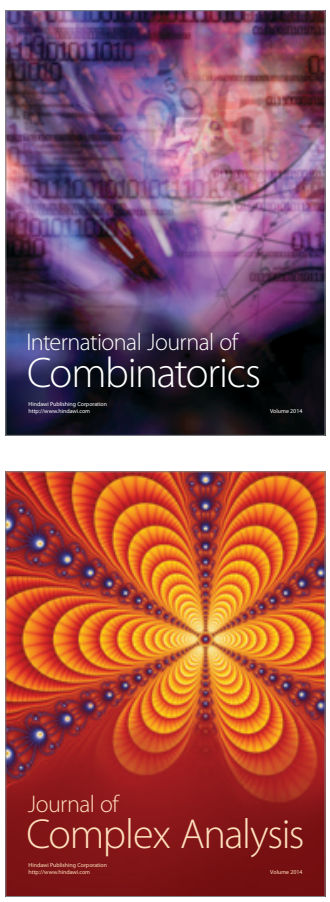

International Journal of

Mathematics and

Mathematical

Sciences
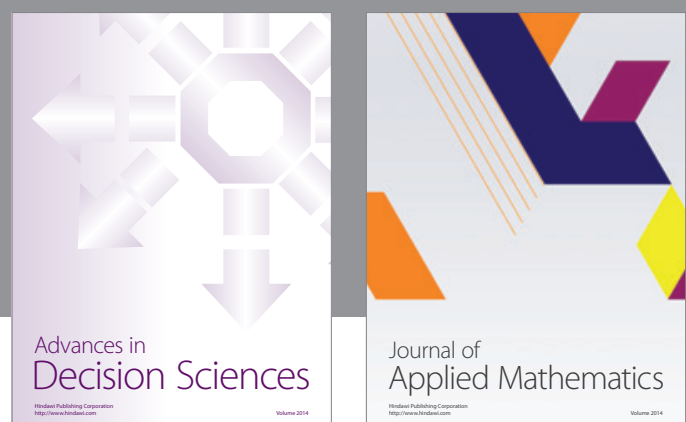

Journal of

Applied Mathematics
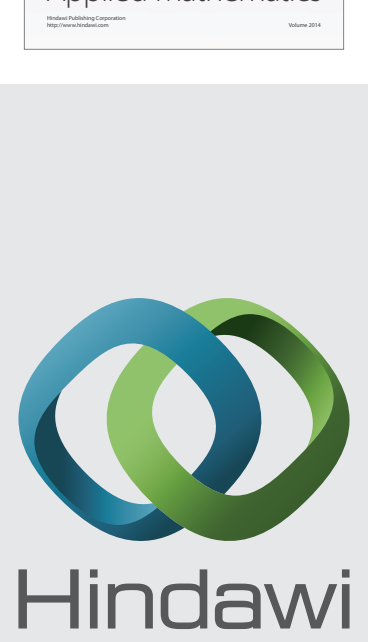

Submit your manuscripts at http://www.hindawi.com
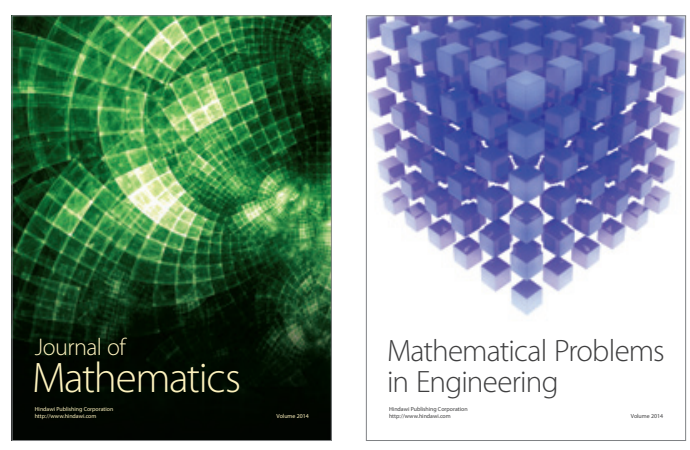

Mathematical Problems in Engineering
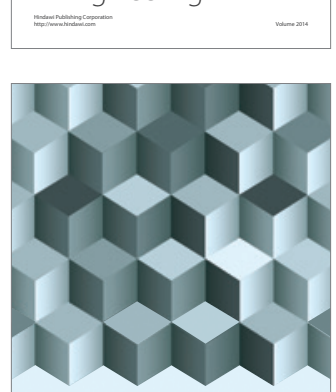

Journal of

Function Spaces
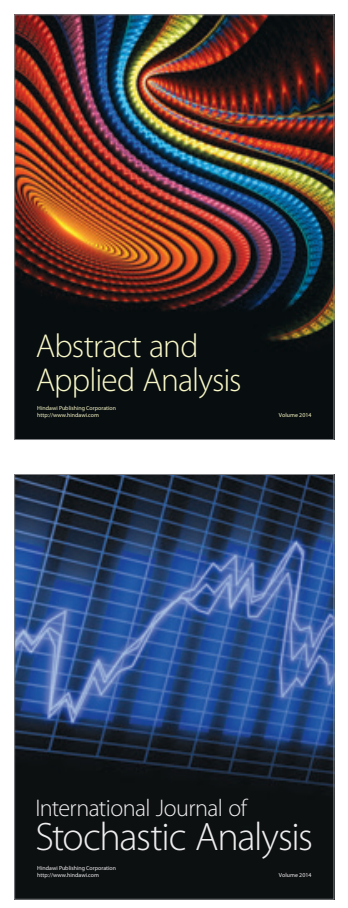

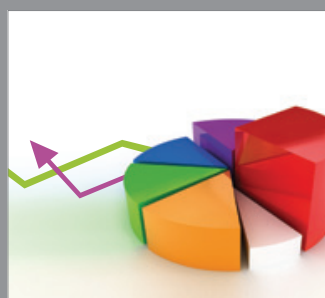

ournal of

Probability and Statistics

Promensencen
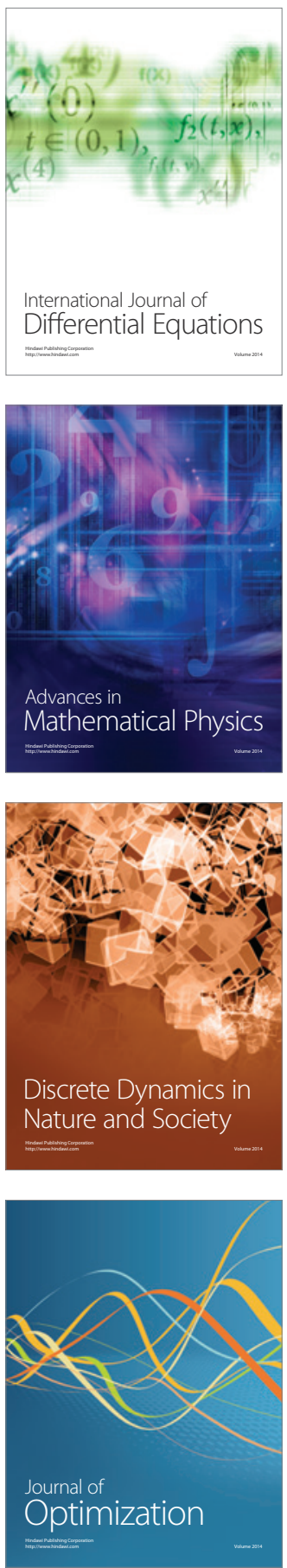